\title{
Bispectrum speckle interferometry of the massive protostellar object S140 IRS 1: Evidence for multiple outflows ${ }^{\star}$
}

\author{
G. Weigelt ${ }^{1}$, Y. Y. Balega ${ }^{2}$, T. Preibisch ${ }^{1}$, D. Schertl ${ }^{1}$, and M. D. Smith ${ }^{3}$ \\ 1 Max-Planck-Institut für Radioastronomie, Auf dem Hügel 69, 53121 Bonn, Germany \\ 2 Special Astrophysical Observatory, Nizhnij Arkhyz, Zelenchuk region, Karachai-Cherkesia, 357147, Russia \\ 3 Armagh Observatory, College Hill, Armagh BT61 9DG, Northern Ireland
}

Received 26 July 2001 / Accepted 18 October 2001

\begin{abstract}
Bispectrum speckle interferometry is employed to explore the immediate environment of the deeply embedded young stellar object S140 IRS 1. A $K^{\prime}$-band $(2.0-2.3 \mu \mathrm{m})$ diffraction-limited resolution of 76 mas $(\sim 70 \mathrm{AU})$ is achieved with the SAO $6 \mathrm{~m}$ telescope, as well as a dynamical range of more than 8 mag and a field of view of $13^{\prime \prime} \times 21^{\prime \prime}$. Our image exhibits many previously unseen complex structures. In addition to the bright, elongated, and very clumpy feature pointing from the central source to the south-east, which was already discussed in a previous paper (Schertl et al. 2000), we find several arc-like structures north-east of IRS 1, extended diffuse emission south of IRS 1, and four new point sources. The diffuse and fragmentary structures close to IRS 1 appear to trace circumstellar material swept up by energetic outflows. In combination with molecular line emission maps from the literature, our image provides direct confirmation that two distinct bipolar outflow systems continue to be driven from IRS 1 on scales between $3^{\prime \prime}$ and $100^{\prime \prime}$. A system of three arc-like structures to the north-east is consistent with cavities excavated by a precessing jet or wind-driven outflow. We discuss the implications for the nature of the central source.
\end{abstract}

Key words. techniques: interferometric - stars: individual: S140 IRS1 - stars: formation

\section{Introduction}

Young massive stars disrupt their surroundings through their intense radiation fields, their high force winds and by their powerful molecular outflows and jets (cf. Garay \& Lizano 1999). An intriguing aspect of massive star formation is the observational result that many massive protostars drive very energetic outflows that hold substantially more mass than the driving protostar (cf. Churchwell 1997; Davis et al. 1998). Evidently, not all of the outflowing material originates from the young stellar object itself; a large fraction seems to be material that is swept up or entrained along the way (Davis et al. 1998). As compared to outflows from low-mass young stellar objects, the outflows from high-mass protostars generally display considerably more complex structure. For example, in molecular line emission maps the outflows often appear to be less well collimated than their low-mass counterparts. Nevertheless, well-collimated jets are associated with some poorly collimated molecular flows (Ray et al. 1990).

Send offprint requests to: G. Weigelt, e-mail: weigelt@mpifr-bonn.mpg.de

* Based based on observations obtained at the Special Astrophysical Observatory with the $6 \mathrm{~m}$ telecope.
In this paper, we present a study of S140 IRS 1, a massive protostellar object with strong outflow activity. Our aim is to investigate how mass is entrained and expelled by resolving the inner section of the outflow region through near-infrared bispectrum speckle interferometry. The rather intricate structure of the molecular outflows from IRS 1, summarized below, makes the immediate circumstellar environment of this object a prime target for a detailed study.

\subsection{Continuum sources in the S140 IRS region}

S140 is an HII region at the southeast edge of the L1204 dark cloud, located at a distance of $\sim 900$ pc (Crampton \& Fisher 1974), which is part of a cloud complex located at the edge of a prominent infrared emission ring, known as the Cepheus ring. About $1^{\prime}$ north-east of the S140 HII region, Rouan et al. (1977) detected strong farinfrared emission at a position at which no objects could be seen in visible light. Beichman et al. (1979) resolved the $20 \mu \mathrm{m}$ infrared emission into three individual sources IRS 1-3 (cf. sketch in Fig. 1). The brightest source, IRS 1, was $7-10$ times brighter than the two other sources. IRS 1 and IRS 3 are bright and very red near-infrared sources 
(Evans et al. 1989 determined $K=6.2, J-K=5.5$ for IRS 1 and $K=7.7, J-K=5.4$ for IRS 3 ), whereas IRS 2 has not been detected in the near-infrared so far. The luminosity of IRS 1 was estimated to be $L \approx 5 \times 10^{3} L_{\odot}$ (Lester et al. 1986), suggesting it to be a deeply embedded ( $A_{V} \approx 30$ mag; Harker et al. 1997) early B-type star with a mass of some $10 M_{\odot}$.

Beichman et al. (1979) detected $6 \mathrm{~cm}$ radio emission from IRS 1 with the VLA. Most of this radio flux probably arises from an optically thin HII region, but a small contribution $(\approx 10 \%)$ seems to originate in a stellar wind (Evans et al. 1989). A few arcseconds from IRS 1, two other radio sources, "VLA4" and "NW", were also detected at $6 \mathrm{~cm}$ (cf. Fig. 1). These two sources, associated with diffuse near-infrared emission, were not detected at $20 \mu \mathrm{m}$. Therefore, Evans et al. (1989) concluded that the radio emission is generated behind strong ionizing shocks where the stellar wind impacts against dense cloud condensations. The wind should blow out a low-density cavity around IRS 1, consistent with the near-infrared polarimetric observations of Yao et al. (1998), who discovered patches of high polarization toward VLA4 and NW, indicating that we observe light scattered from IRS 1.

In the dense cloud core south-west of IRS 1, Minchin et al. (1995) discovered two submillimetre sources about $\sim 15^{\prime \prime}$ to the west and south-west of IRS 1 , respectively, and a third $\sim 20^{\prime \prime}$ north-east of IRS 1 (see Fig. 1 ). These sources, for which so far no infrared counterparts could be found, may be deeply embedded protostars in a very early evolutionary stage.

\subsection{Outflows in the $S 140$ IRS region}

A molecular CO outflow from IRS 1 was first detected by Blair et al. (1978). Bally \& Lada (1983) calculated a total outflow mass of $\sim 64 M_{\odot}$, which is $\sim 6 \times$ higher than the estimated mass of the protostar IRS 1 . They estimated a mechanical power of the outflow of $\sim 90 L_{\odot}$, an outflow velocity of $\sim 20 \mathrm{~km} \mathrm{~s}^{-1}$, and a mass loss rate of $\sim 0.003 M_{\odot} / \mathrm{yr}$. Minchin et al. (1993) studied the CO molecular line emission in the S140 region with the JCMT and found position angles ${ }^{1}$ of $\sim 160^{\circ}$ and $\sim 340^{\circ}$ for the blue- and red-shifted outflow components, respectively. We will call this bipolar outflow structure the $160^{\circ} / 340^{\circ}$ outflow in the following text. Since the outflow lobes overlap strongly, they concluded that the outflow axis must be closer to the line of sight than to the plane of the sky. Their CO map also indicated the presence of low-velocity blue-shifted CO emission north-east of IRS 1 , at a position angle of $\sim 20^{\circ}$.

Molecular CS emission was mapped by Hayashi \& Murata (1992). They found a prominent $\mathrm{V}$-shaped ridge (radius $\sim 0.7^{\prime}$ ) of dense material around S140 IRS, in which the north-eastern part is missing and proposed that this half-ring might be the remnant of the cloud core from which the S140 IRS cluster has formed. They also obtained

\footnotetext{
1 All position angles are measured in counter-clock direction from north.
}

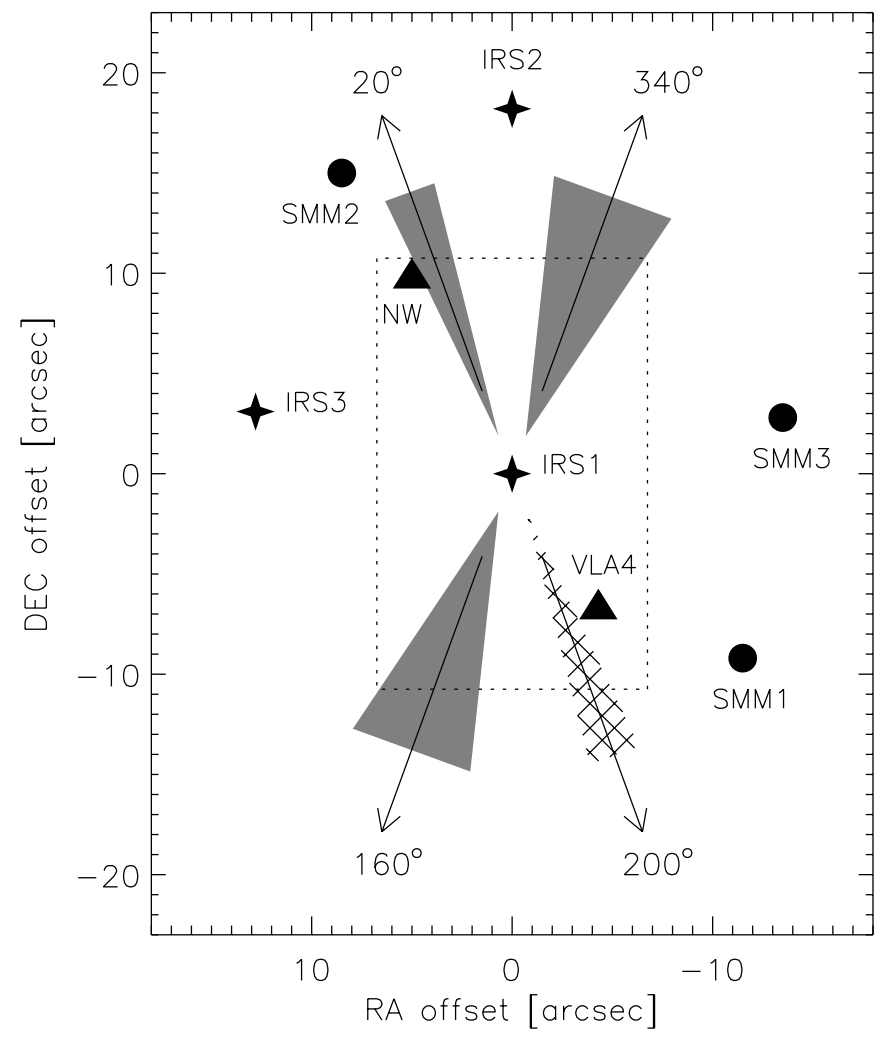

Fig. 1. Schematic map of the S140 region. The asterisks mark the positions of the infrared sources, the dots those of the submm peaks, the triangles those of the two radio continuum sources. Outflow directions are indicated by arrows superposed on shaded triangles; the widths of the triangles indicate the strength of the molecular line emission of the outflow lobes. The hatched triangle marks the direction of the $200^{\circ}$ outflow, for which the molecular emission maps provide only weak evidence. The dotted-lined box marks the field of view of our new speckle image. North is up and east is to the left.

a CO map and again found clear evidence for a bipolar outflow in the $160^{\circ} / 340^{\circ}$ direction. Their CO map again showed blue-shifted molecular emission north-east of IRS 1 and a weak elongation of the blue-shifted lobe in the south-west direction at a position angle of $\sim 205^{\circ}$.

The outflow has been mapped in $\mathrm{HCO}^{+}$by Wilner \& Welch (1994). The red-shifted emission lobe is strongest north-east of IRS 1 at a position angle of $\sim 15^{\circ}$. The position angle of this feature agrees reasonably well with that of the north-eastern $\left(20^{\circ}\right) \mathrm{CO}$ component found in the data of Hayashi \& Murata (1992) and Minchin et al. (1993). Their maps also showed a weak elongation of the blue-shifted emission in the south-west direction.

The existence of a quadrupolar outflow from IRS 1 was proposed by Yao et al. (1998) and supported by nearinfrared polarimetric images. A sketch of the region showing the location of the sources and the directions of the outflow lobes is shown in Fig. 1. The radio data show that molecular material seems to be flowing away from IRS 1 in at least three different directions. The main structure is the $160^{\circ} / 340^{\circ}$ bipolar outflow. Despite the apparently 
high degree of symmetry of this bipolar outflow, Minchin et al. (1993) noted that the mass within the blue-shifted (south-eastern) outflow lobe is $\sim 2.5$ times the mass in the redshifted (north-western) lobe. Another outflow lobe, which we will call the $20^{\circ}$ lobe, is oriented in the northeast direction. The molecular line maps also indicate the possible existence of a weak low-velocity outflow lobe in the $\sim 200^{\circ}$ direction which might be the counterpart of the $20^{\circ}$ lobe.

First high-resolution infrared observations of IRS 1 were obtained by Hoare et al. (1996) and revealed diffuse emission extending to the south-east of the source. In a previous paper (Schertl et al. 2000), we presented a bispectrum speckle interferometric image and polarimetric measurement of the central $3^{\prime \prime} \times 3^{\prime \prime}$ region around IRS 1 . This image showed a bright elongated clumpy structure pointing away from the central source with a position angle of $154^{\circ} \pm 3^{\circ}$, consistent with the direction of the blueshifted lobe of the $160^{\circ} / 340^{\circ}$ outflow. The centrosymmetric pattern of high polarization of this feature indicates that we see scattered light from the central source. We present here further high-resolution infrared speckle observations. The data in this paper cover a field of about $13^{\prime \prime} \times 21^{\prime \prime}$, i.e. an area 11 times larger than in our previous study.

\section{Observations and data reductions}

The speckle interferograms were obtained with the $6 \mathrm{~m}$ telescope at the Special Astrophysical Observatory (SAO) in Russia and our Hawaii array speckle camera on 19 and 24 September 1999. The observations were made in the $K^{\prime}$-band (central wavelength of the filter $2.165 \mu \mathrm{m}$, $F W H M$ bandwidth $0.328 \mu \mathrm{m}$ ). The exposure time per frame was $150 \mathrm{~ms}$, the pixel size was 27.0 mas and seeing was $\sim 1^{\prime \prime}$. A total number of 6683 speckle interferograms of S140 IRS 1 and 6635 speckle interferograms of reference stars (HIP 110410 and HIP 110498) were taken. The reconstructed S140 IRS 1 image presented in Fig. 2 is a $13^{\prime \prime} \times 21^{\prime \prime}$ mosaic derived from the following three images:

(a) a $6.9^{\prime \prime} \times 6.9^{\prime \prime}$ image centered on the bright central source of S140 IRS 1 (reconstruction from the 5552 speckle frames of S140 reported in Schertl et al. 2000; $256^{2}$ pixels per frame),

(b) a $13.8^{\prime \prime} \times 13.8^{\prime \prime}$ image centered on a position approx. $3.2^{\prime \prime}$ south of the central source (reconstruction from 435 S140 speckle frames of $512^{2}$ pixels per frame),

(c) a $13.8^{\prime \prime} \times 13.8^{\prime \prime}$ image centered on a position approx. 5. $6^{\prime \prime}$ north of the central source (reconstruction from 696 S140 speckle frames of $512^{2}$ pixels per frame).

The images were reconstructed using the bispectrum speckle interferometry method (Weigelt 1977; Lohmann et al. 1983; Weigelt et al. 1991). The bispectra of the frames consisted of up to 200 million elements. The modulus of the object Fourier transform was determined with the speckle interferometry method (Labeyrie 1970). The three sub-images were aligned by centering on the bright point-like source IRS 1 and then added with equal weights. The resulting image (Fig. 2) has a diffraction-limited resolution $\lambda / D$ of 76 mas.

\section{Morphology in the $K^{\prime}$-band image}

The reconstructed image of S140 IRS 1 is displayed in Fig. 2. The large-scale structure in our image corresponds extremely well with direct $K$-band images of lower resolution (e.g. Harker et al. 1997; Yao et al. 1998) as well as our previous image of the central region (Schertl et al. 2000).

The prominent features are labelled in Fig. 3. Our field of view does not include the infrared sources IRS 2 and IRS 3, but includes the locations of the radio sources VLA4 and NW. Since these two radio sources have no infrared counterparts, we have derived their positions relative to IRS 1 from the coordinates given by Evans et al. (1989). The features labelled in Fig. 3 are as follows:

- bright extended emission pointing from IRS 1 towards the south-east, as discussed in detail by Schertl et al. (2000). The central features A-F are marked in our image. The structure of the emission near IRS 1 is very clumpy, and its general shape follows a well-defined direction with a position angle of $154^{\circ} \pm 3^{\circ}$;

- very inhomogeneous and clumpy large-scale diffuse emission $(\mathrm{H})$ to the south of IRS 1 ;

- a prominent arc of emission (I) approximately $10^{\prime \prime}$ northeast of IRS 1 . This feature has a bow-shock like shape and appears to point away from IRS 1 at a position angle of $20^{\circ}$ (cf. Fig. 3). The bright apex of the arc appears to be connected with IRS 1 by faint emission;

- an additional, fainter, arc-like feature $(\mathrm{J})$ can be seen immediately to the west of the $20^{\circ}$ arc. The position angle of its axis is approximately $10^{\circ}$. This arc also seems to be connected with IRS 1 by faint emission;

- yet another arc-like feature $(\mathrm{K})$ with a position angle of approximately $25^{\circ}$ is found to the north-east of the $20^{\circ}$ arc;

- a bright arc of emission (L) can be seen $\sim 3.5^{\prime \prime}$ south of IRS 1;

- a wiggly feature (M) showing an "inverse S" shape can be seen $\sim 5^{\prime \prime}$ south-east of IRS 1 . This feature seems to be connected with arc $\mathrm{L}$. We note that features $\mathrm{M}$ and $\mathrm{L}$ seem to form a wave-like structure, and that a similar wave-structure can be seen $\sim 3^{\prime \prime}$ below;

- a bright isolated knot $(\mathrm{N})$ is located $\sim 3.5^{\prime \prime}$ north-east of IRS 1;

- our image also reveals four previously unknown pointlike sources. We will denote these sources with the names IRS 4 to IRS 7. IRS 4 is located $3.9^{\prime \prime}$ south-west of IRS 1. This source is $4.2 \pm 0.2 \mathrm{mag}$ fainter than IRS 1 in the $K^{\prime}$ band. IRS 5 is seen $7.3^{\prime \prime}$ north-east of IRS 1 . This source is $4.8 \pm 0.2 \mathrm{mag}$ fainter than IRS 1 , and it appears to be connected to the arcs by an arc of diffuse emission. IRS 6 is located $5.3^{\prime \prime}$ north-west of IRS 1 and $7.4 \pm 0.2$ mag fainter than IRS 1. IRS 7 is seen $4.0^{\prime \prime}$ north of IRS 1 and is $8.0 \pm$ 0.2 mag fainter than IRS 1. 


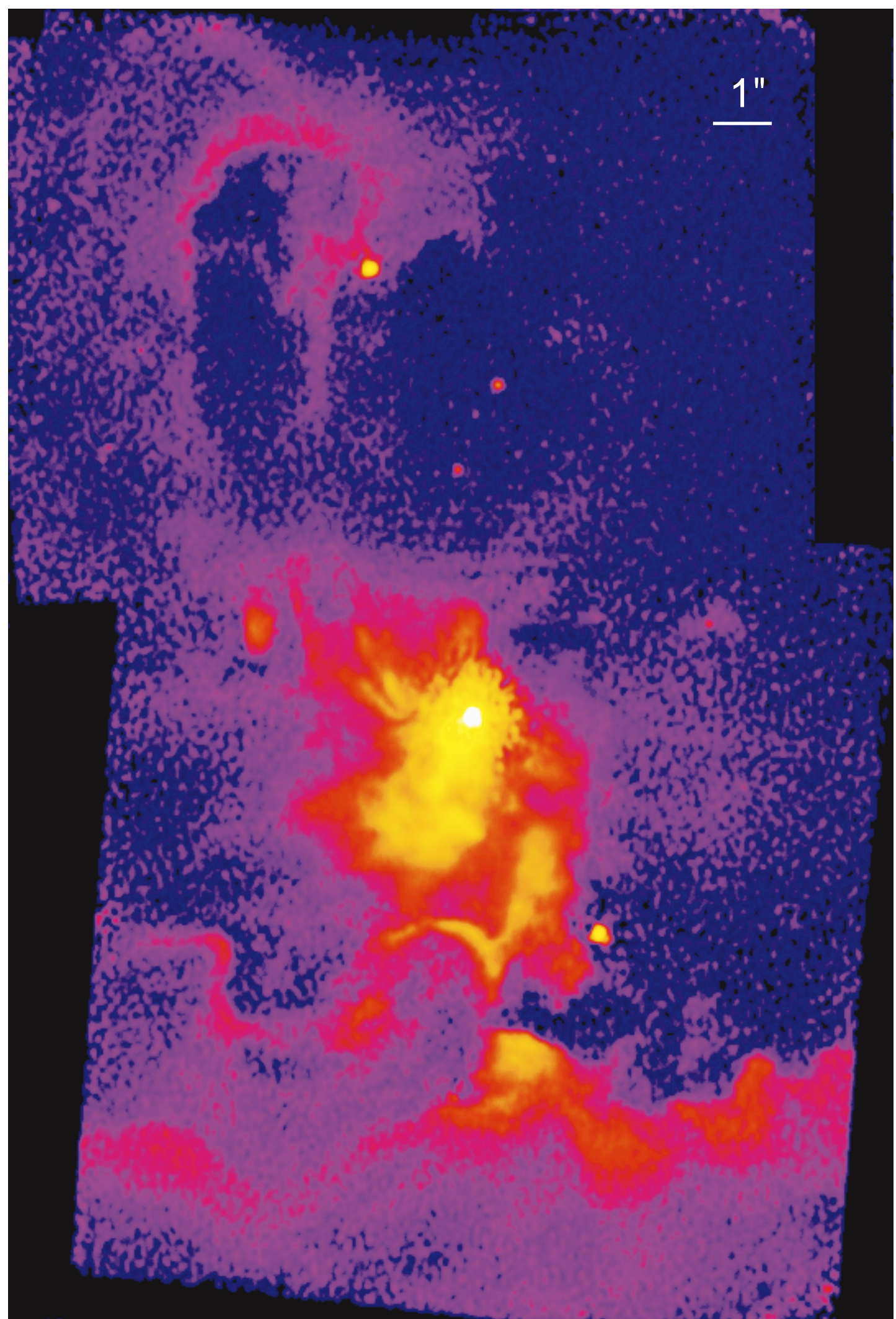

Fig. 2. Pseudocolor representation of our $K^{\prime}$-band mosaic image of a $13^{\prime \prime} \times 21^{\prime \prime}$ area centered on S140 IRS 1 , reconstructed by the bispectrum speckle interferometry method. North is up and east is to the left. The image has a diffraction-limited resolution of 76 mas. The intensity scale is based on a square-root relation and was adjusted to show both the central bright as well as the faint arc-like structures with good contrast. The faintest structures visible in the image are 8-9 mag fainter than the central source IRS 1 (for example, the point source $4^{\prime \prime}$ north of IRS 1 is $\sim 8.0$ mag fainter than the central source IRS 1 ). The photometric accuracy is approximately $\pm 0.2 \mathrm{mag}$. 


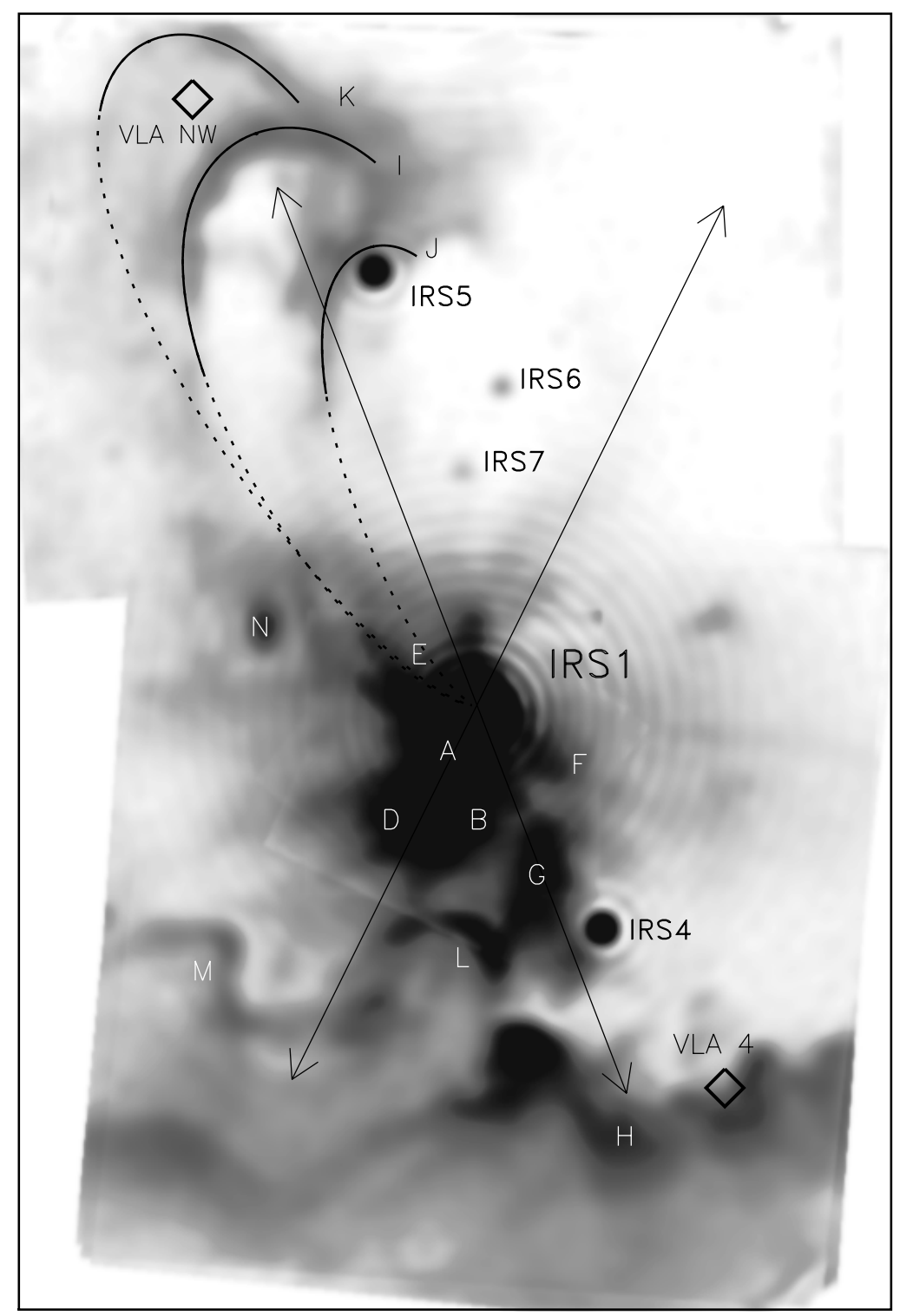

Fig. 3. Greyscale representation of our $K^{\prime}$-band mosaic of S140 IRS 1 reconstructed with a reduced resolution of 240 mas in order to show more clearly the fainter details. The intensity scale is based on a square-root relation and was adjusted to show both the central bright as well as the faint arc-like structures with good contrast. The features discussed in the text are marked in this image. The curved lines show the shapes of the cavity models described in Sect. 4.2. The rhombs mark the positions of the VLA sources 4 and NW. The long arrows indicate the four major outflow directions. North is up and east is to the left.

\section{Interpretation of the observed structures}

\subsection{Central and south-east region}

In Schertl et al. (2000), we suggested that the bright extended structure pointing from IRS 1 to the south-east is the partially evacuated cavity in the circumstellar envelope around IRS 1 , excavated by the blue-shifted southeastern $\left(160^{\circ}\right)$ molecular outflow lobe. Our new image again demonstrates that the cavity appears to be very clumpy. The clumpiness may be an indication of the presence of individual bullets or unresolved bow shocks in a jet that drives the outflow. Features $\mathrm{M}$ and D suggests that some radiation from IRS 1 does find a path through the high extinction envelope of IRS 1 . No infrared emission is seen in our image in the direction of the red-shifted $\left(340^{\circ}\right)$ CO outflow lobe, probably due to high extinction in the north-western part of the circumstellar material around IRS 1.

\subsection{North-eastern region}

Three wide arc-like structures dominate the north-eastern part of our image. The brightest arc (I) has an orientation (defined as the position angle of the line connecting IRS 1 with the tip of the arc) of $20^{\circ}$. All three arcs point away from IRS 1 . The shapes of their tips, especially that of the $20^{\circ}$ arc, are similar to bow shocks. These arcs appear to trace the interaction of material flowing away from IRS 1 
with the surrounding medium. The $20^{\circ}$ arc appears to be connected to IRS 1 by a faint curved extension on its eastern side. It might be directly connected with feature E. The $10^{\circ}(\mathrm{J})$ and the $25^{\circ}(\mathrm{K})$ arcs might have similar extensions towards IRS 1 at their eastern sides, however, these features are too faint to be clearly visible in our images.

The general shapes of these arcs show remarkable similarity to outflow cavities. We will therefore compare the observed shapes with theoretically expected morphologies of outflow cavities based on the model for propagating outflows presented by Gueth \& Guilloteau (1999). These authors presented semi-analytical models of jet-driven flows, in which prompt entrainment occurs at the head of the traveling bow shock. They could reproduce with their model the observed shape of the cavity related to the extremely young HH 211 jet very well. For the bow shape they use the analytical solution for the geometry of a momentum-driven bow shock from Wilkin (1996) which, in cylindrical coordinates $\left(R_{\mathrm{c}}, \theta\right)$, is given by

$$
R_{\mathrm{c}}(\theta)=R_{0} \sqrt{3\left(1-\frac{\theta}{\tan \theta}\right)} .
$$

As the bow shock propagates along the jet axis $x$, its size $R_{0}$ is assumed to vary according to the relation

$R_{0}(x)=R_{0}(d)\left(\frac{x}{d}\right)^{-\gamma / 2}$,

where $d$ is the current distance from the jet source. The exponent $\gamma$ depends on the density distribution in the ambient cloud. For a protostellar envelope where the density decreases as $x^{-2}$ one finds $\gamma=-2$. Finally, the projection on the jet axis is given by

$x=d+\frac{R_{\mathrm{c}}(\theta)}{\tan \theta}$.

Projected shapes computed with this model are displayed in Fig. 3 for the case $\gamma=-2$, overlaid on the three arcs. The parameters we used to compute these models are $d=10.80^{\prime \prime}(9720 \mathrm{AU}), R_{0}(d)=1.03^{\prime \prime}(920 \mathrm{AU})$ for the $20^{\circ}$ arc $, d=8.64^{\prime \prime}(7780 \mathrm{AU}), R_{0}(d)=0.52^{\prime \prime}$ (470 AU) for the $10^{\circ}$ arc, and $d=13.50^{\prime \prime}(12150 \mathrm{AU}), R_{0}(d)=0.81^{\prime \prime}$ (730 AU) for the $25^{\circ}$ arc.

The model fits the observed arc structures in our image quite well. This suggests that the observed arcs actually represent the limb-brightened flanks of elongated cavities that have probably been carved out by material flowing away from IRS 1. For all three cavities only the eastern flank can be seen, while the western flanks are either absent or much fainter. One reason for this might be that the individual cavities are seen projected onto each other, i.e. the $20^{\circ}$ cavity hides some part of the western flank of the $25^{\circ}$ cavity, and the $10^{\circ}$ cavity hides some part of the western flank of the $20^{\circ}$ cavity. Another reason may be that the three cavities are interacting and therefore their shapes are somewhat disturbed.

The orientation of these arcs closely corresponds to the direction of the blue-shifted $\mathrm{CO}$ emission north-east of IRS 1 , at a position angle of $\sim 20^{\circ}$. The molecular line emission shows that material is actually flowing away from IRS 1 in a north-eastern direction. Further support for the assumption of an outflow in the $\sim 20^{\circ}$ direction comes from the location of the strong radio source "NW" detected with the VLA, which is found just between the $25^{\circ}$ arc and the $20^{\circ}$ arc. As this radio source has no infrared counterpart, its emission probably originates from ionized shocks that are created by the collision of out-flowing material with surrounding material (Evans et al. 1989).

\subsection{Southern part}

The southern part of our image is dominated by clumpy diffuse emission. The CS emission map of Hayashi \& Murata (1992) shows dense clumps of molecular cloud material in a ring-like structure to the south of IRS 1 . In the $800 \mu \mathrm{m}$ emission map of Minchin et al. (1995) the peak of the emission is about $15^{\prime \prime}$ south-west of IRS 1 . We therefore believe that the clumpy diffuse emission we see in our image approximately $7^{\prime \prime}-10^{\prime \prime}$ south and south-west of IRS 1, comes from the surface of this molecular cloud clump. The high degree of near-infrared polarization in that region found by Yao et al. (1998), suggests that some part of the emission from theses features is light originating from IRS 1 and being reflected at the surface of the cloud clump.

Note that the surface of the diffuse emission seems to show some kind of depression next to the feature $\mathrm{H}$ in Fig. 2. This structure could indicate the impact of material flowing from IRS 1 in the $\sim 200^{\circ}$ direction. This is just the opposite direction to the symmetry axis of the northeastern $20^{\circ}$ arc. The location of the radio source VLA 4 near that feature is a further indication that out-flowing material from IRS 1 collides with cloud material in this direction.

Other prominent features in this region are the bright $\operatorname{arcs} \mathrm{L}$ and G. It appears likely that these arcs represent the limb-brightened upper surfaces of dense clumps, swept up by a south-west outflow, that reflect light from IRS 1 . We note that the apparent depression of the wave-like structure extending from feature $\mathrm{M}$ to feature $\mathrm{L}$ (and also in the similar wave-like structure $\sim 3^{\prime \prime}$ below) might be related to the $160^{\circ}$ outflow.

The true nature of the complex diffuse structures in this part of the image remains unclear, because the morphology is determined by several factors: First, the surface of the cloud can be expected to show intrinsic clumpiness. Second, the surface is most likely distorted by outflowing material, probably by individual bullets moving in variable directions. Third, these structures are illuminated with light from IRS1 escaping through the $160^{\circ}$ cavity, producing a complex illumination pattern. Forth, one also has to consider geometrical projection effects.

This region also contains the point-like source IRS 4 . It is another embedded young stellar object or perhaps a background star. 


\section{Discussion}

Our image clearly traces the ongoing violent interaction of material flowing away from IRS 1 with the surrounding cloud material in several directions. The most prominent features of diffuse emission trace the south-eastern part of the previously known $160^{\circ} / 340^{\circ}$ molecular outflow. The system of arcs north-east of IRS 1 suggests an additional flow of material in the $\sim 20^{\circ}$ direction. This is supported by the presence of the radio source VLA NW associated with these arcs. The fact that these features trace exactly the direction of the low-velocity wing in the $\mathrm{CO}$ and $\mathrm{HCO}^{+}$ emission maps (see Sect. 1.2) strongly supports our assumption that the arcs are related to the larger scale outflow activity in the $\sim 20^{\circ}$ direction. It is also worth remarking that this direction coincides with the direction in which the dense molecular ring around S140 IRS is broken (cf. Hayashi \& Murata 1992). This suggests that the outflowing material has broken and cleared the ring in this direction.

There is also some evidence, although less convincing, for recent flow of material in the opposite, i.e. $200^{\circ}$, direction: the apparent depression in the surface of the cloud core near feature $\mathrm{H}$, the location of the radio source VLA 4, and the extension of the low-velocity CO emission in that direction.

Yao et al. (1998) concluded that a quadrupolar outflow would help explain the large scale near-infrared reflection nebulae around IRS 1. Taken together, the data suggest the presence of two ongoing and distinct bipolar outflow systems, both originating from IRS 1 . The projected angle between these two bipolar flows is $\sim 45^{\circ}$. For the $160^{\circ} / 340^{\circ}$ molecular outflow, the red- and blue-shifted CO lobes are known to overlap strongly, which suggests that the outflow axis must be closer to the line-of-sight than to the plane of the sky. This is consistent with the structure of the diffuse near-infrared emission in our image: we see only the cavity of the $160^{\circ}$ flow, but no indication for any structure in the $340^{\circ}$ direction, which suggests that the outflow structure is tilted towards the line-of-sight and that we look "into" the southern cavity. We do not see the $340^{\circ}$ cavity, because it is oriented away from us and, furthermore, is hidden by circumstellar extinction. The axis of the $20^{\circ} / 200^{\circ}$ outflow, however, seems to be rather close to the plane of the sky. One reason for this assumption is that this outflow is seen only in low-velocity molecular line emission.

In order to achieve more insight into this complex object, there are two main questions we have to explain:

1: How can we understand the presence of a quadrupolar outflow from IRS 1 ?

2: How can we explain the system of arcs north-east of IRS 1 ?

\subsection{Multiple outflow directions}

Many explanations have been proposed for multiple outflow directions: multiple protostars driving individual outflows (cf. Reipurth et al. 2000), precession due to orbital motion of a binary (Fendt \& Zinnecker 1998), jet precession and wiggling due to disk precession and wobbling (cf. Terquem et al. 1999; Bate et al. 2000), funneling of a wind through multiple nozzles (e.g. Norman et al. 1981; Königl 1982), fluid dynamical instabilities in swept up shells (Stone et al. 1995), deflection from external clouds and clumps (e.g. de Gouveia Dal Pino 1999), misaligned stellar and disk winds.

The evidence for two bipolar outflow directions on both small and large scales implies that scenarios involving funneling, fluid instabilities and deflection are implausible. An evolving multiple-protostar system with a precessing twin-outflow engine or two separate outflows remains in consideration. The most straightforward explanation for the presence of two distinct bipolar outflow structures, the $160^{\circ} / 340^{\circ}$ and the $20^{\circ} / 200^{\circ}$ outflow, would be that we see two independent outflows from two sources, i.e. that IRS 1 is a binary or multiple system. We note that several other examples of multipolar jets and outflows have recently been found, and in many cases their energy source has been found to be multiple. For example, Reipurth et al. (1999) found evidence that the HH 111 energy source drives a quadrupolar flow. In $3.6 \mathrm{~cm}$ radio images they detected a binary companion to the central source at a separation of less than $50 \mathrm{AU}$, suggesting that each of the binary components drives its own bipolar flow. Another example was reported by Fridlund \& Liseau (1998) and Itoh et al. (2000), who detected two independent twisted jets emanating from L1551 IRS 5. Since L1551 IRS 5 is known to be a young binary system separated by $\sim 40 \mathrm{AU}$ and embedded in a circumbinary disk, they assume that each of the binary components drives one of the jets.

In the case of S140 IRS 1, it is interesting to note that in our analysis of the central image (Schertl et al. 2000) we found marginal evidence that S140 IRS 1 is slightly extended with a FWHM diameter of approximately 20 mas. This might indicate that the central source is multiple and in that case would suggest a separation of $\sim 20 \mathrm{AU}$. Another possibility would be that there is a further outflow source near IRS 1 that we do not see in our image because it is either too deeply embedded or intrinsically much fainter than IRS 1.

\subsection{Origin of the north-eastern cavities}

The observed cavities have probably been created by a jetdriven outflow. As the jet plows through the gas and dust in the circumstellar environment of the protostar, it produces shock waves that heat the gas. The strongest shock is expected at the tip of the jet (bow shock), weaker shocks at the edges where the hot and dense matter of the jet is expanding sidewards. The entrainment and deflection of ambient gas by the bow shock generates a wide cavity. Hence, sharp features at the edges of the cavities originate mostly from hot shocked material while radiation from the central object is reflected from the cavity walls. This expectation is consistent with the observed shapes of 
the arcs in our image, as we have demonstrated by fitting their morphologies with the predicted cavity shapes of the jet-driven outflow model. The fact that we see three individual cavities can be explained by a discontinuous jet consisting of a series of individual bullets.

It is worth noting that the projected distances of the tips of the arcs are increasing with their respective position angles. This morphology suggests that the direction of the individual flows that have created the individual cavities has changed with time. In the jet-driven scenario, this is an indication for jet precession. We note that theoretical simulations of outflows driven by pulsating jets (e.g. the model for so-called Hammer jets presented by Völker et al. 1999) can reproduce the general appearance of the complex of arcs in our image quite well. The fact that we see only three individual arcs does not mean that there were only three ejection events, because, first, there might be more features just outside the field of view of our image, and, second, bow shocks are transient features that are visible only for a limited period of time. This model also offers an interesting explanation for the fact that the eastern parts of the arcs can be seen much better than the western parts: each flow might have disturbed and perhaps destroyed the western part of the cavity of the previous flow.

If we would know the outflow velocity, we could compute the kinematical age of the flows associated with the individual arcs and from this infer the amount of time between the ejection events that created the individual arcs. The velocity of the molecular gas in the extended outflow $160^{\circ} / 340^{\circ}$ has been determined to be $\sim 20 \mathrm{~km} \mathrm{~s}^{-1}$ by Bally \& Lada (1983). This value, however, probably refers to surrounding gas that is swept away by the force of the outflow and can presumably only be considered as a lower limit for the outflow velocity. The observed shape of the arcs suggests that the $20^{\circ}$ outflow is jet-driven, and the velocity of the jet is likely to be significantly higher than this. A reasonable value for the flow velocities in jets is 100 $200 \mathrm{~km} \mathrm{~s}^{-1}$ (cf. Eislöffel et al. 2000; Richer et al. 2000). Using this assumption, the kinematic ages of the three jets (i.e. their projected length divided by the assumed jet velocity) would be 160-320 years, 200-400 years, and 270-540 years, respectively. This implies that the outflow direction has changed by $\sim 15^{\circ}$ within $\sim 110-220$ years.

What makes these numbers very interesting, is the fact that the expected precession time scale in non-coplanar close binary systems lies in the same range. Assuming that S140 IRS 1 actually is a binary with a separation of $\sim 20 \mathrm{AU}$ and a mass of $\sim 10 M_{\odot}$ (see above), the Keplerian orbital period for such a system would be about 30 years. Several theoretical studies (e.g. Papaloizou \& Terquem 1995; Terquem 1998; Bate et al. 2000) have considered the dynamics of a protostellar disk in a binary system where the disk is misaligned with the orbital plane of the binary. It was found that in such a system the disk (and therefore also the jet) precesses with a period of the order of 20 binary periods. For the (still hypothetical) S140 IRS 1 binary system one therefore would expect a jet precession period of $\sim 600$ years. Since the observed structure of the north-eastern complex of arcs actually suggests changes of the outflow axis direction on time scales of a few hundred years, this model may be a good explanation of the S140 IRS 1 jet.

\section{Summary and conclusions}

The two main results of our study are as follows:

(1) S140 IRS 1 appears to be the source of two individual bipolar outflow structures on the scale of a few arcsec as well as on scales of up to 100 arcsec. Besides the previously well known $160^{\circ} / 340^{\circ}$ outflow, we find evidence of material flowing out from IRS 1 in the $20^{\circ} / 200^{\circ}$ direction.

(2) In the $20^{\circ}$ outflow lobe we find a complex of three arcs with a systematic positional offset of their bowshaped tips. The position of the arcs corresponds to the approximate location of a radio source and polarised infrared knot. This structure could be well explained as the successive sites of impact of a precessing jet-driven outflow and cavity.

These results can be understood in a scenario assuming that S140 IRS 1 is a close binary system in which the disks are misaligned with the orbital plane of the system. In this scenario, each star would be responsible for one of the outflows. The precessing outflow that created the three individual cavities in the $20^{\circ}$ lobe would then be due to precession of the non-coplanar disk.

Our results clearly demonstrate that S140 IRS 1 has a very complex environment and deserves further observational efforts. In particular, proper motion measurements of the near environment will answer many of the dynamical questions. An attempt to resolve the suspected close binary system (expected separation $\$ 20$ mas) should soon be possible with long-baseline interferometry.

\section{References}

Bally, J., \& Lada, C. J. 1983, ApJ, 265, 824

Bate, M. R., Bonnell, I. A., Clarke, C. J., et al. 2000, MNRAS, 317,773

Beichman, C. A., Becklin, E. E., \& Wynn-Williams, C. G. 1979, ApJ, 232, L47

Blair, G. N., Evans, N. J. II, Vandenbout, P. A., \& Petters, W. L. III 1978, ApJ, 219, 896

Brooke, T. Y., Sellgren, K., \& Smith, R. G. 1996, ApJ, 459, 209

Churchwell, E. 1997, ApJ, 479, L59

Crampton, D., \& Fisher, W. A. 1974, Pub. Dom. Ap. Obs., 14, 12

Davis, C. J., Moriarty-Schieven, G., Eislöffel, J., Hoare, M. G., \& Ray, T. P. 1998, ApJ, 115, 1118

Eislöffel, J., Mundt, R., Ray, T. P., \& Rodriguez, L. F. 2000, in Protostars and Planets IV, ed. V., Mannings, A. P., Boss, \& S. S., Russel (Tucson: University of Arizona Press), 815

Evans, N. J. II, Mundy, L. G., Kutner, M. L., \& DePoy, D. L. 1989, ApJ, 346, 212

Fendt, C., \& Zinnecker, H. 1998, A\&A, 334, 750 
Fridlund, C. V. M., \& Liseau, R. 1998, APJ, 499, L75

Garay, G., \& Lizano, S. 1999, PASP, 111, 1049

de Gouveia Dal Pino, E. M. 1999, ApJ, 526, 862

Gueth, F., \& Guilloteau, S. 1999, A\&A, 343, 571

Harker, D., Bregman, J., Tielens, A. G. G. M., Temi, P., \& Rank, D. 1997, A\&A, 324, 629

Hayashi, M., \& Murata, Y. 1992, PASJ, 44, 391

Hoare, M. G., Glindemann, A., \& Richichi, A. 1996, in The Role of Dust in the Formation of Stars, ed. H. U., Käuf, \& R., Siebenmorgen (Berlin: Springer), 35

Itoh, Y., Kaifu, N., Hayashi, M., et al. 2000, PASJ, 52, 81

Königl, A. 1982, ApJ, 261, 115

Labeyrie, C. D. 1970, A\&A, 6, 85

Lester, D. F., Harvey, P. M., Joy, M., \& Ellis, H. B. Jr. 1986, ApJ, 309, 80

Lohmann, A. W., Weigelt, G., \& Wirnitzer, B. 1983, Appl. Opt., 22, 4028

Minchin, N. R., White, G. J., \& Padman, R. 1993, A\&A, 277, 595

Minchin, N. R., Ward-Thompson, D., \& White, G. J. 1995, A\&A, 298, 894

Norman, M. L., Smarr, L., Smith, M. D., \& Wilson, J. R. 1981, ApJ, 247, 52

Papaloizou, J. C. B., \& Terquem, C. 1995, MNRAS, 274, 987

Ray, T. P., Poetzel, R., Solf, J., \& Mundt, R. 1990, ApJ, 357, L45
Reipurth, B., Yu, K. C., Rodríguez, L. F., Heathcote, S., \& Bally, J. 1999, A\&A, 352, L83

Reipurth, B., Yu, K. C., Heathcote, S., Bally, J., \& Rodríguez, L. F. 2000, AJ, 120, 1449

Richer, J., Shepherd, D., Cabrit, S., Bachiller, R., \& Churchwell, E. 2000, in Protostars and Planets IV, ed. V., Mannings, A. P., Boss, \& S. S., Russel (Tucson: University of Arizona Press), 867

Rouan, D., Lena, P. J., Puget, J. L., de Boer, K. S., \& Wijnbergen, J. J. 1977, ApJ, 206, L35

Schertl, D., Balega, Y., Hannemann, T., et al. 2000, A\&A, 361, L29

Smith, M. D., Suttner, G., \& Yorke, H. W. 1997, A\&A, 323, 223

Stone, J. M., Xu, J., \& Mundy, L. G. 1995, Nature, 377, 315

Terquem, C. 1998, ApJ, 509, 819

Terquem, C., Eislöffel, J., Papaloizou, J. C. B., \& Nelson, R. P. 1999, ApJ, 512, L131

Völker, R., Smith, M. D., Suttner, G., \& Yorke, H. W. 1999, A\&A, 343, 953

Weigelt, G. 1977, Opt. Commun., 21, 55

Weigelt, G. 1991, in Progress in Optics, 29, ed. E. Wolf (Elsevier Science Publishers), 295

Wilkin, F. P. 1996, ApJ, 459, L31

Wilner, D. J., \& Welch, W. J. 1994, ApJ, 427, 898

Yao, Y., Ishi, M., Nagata, T., et al. 1998, ApJ, 500, 320 